\title{
Superposición restringida de imágenes
}

\begin{abstract}
RESUMEN
Se presenta un algoritmo para seleccionar un conjunto de píxeles por encima de un cierto nivel de gris en una imagen, extraerlo y transferirlo a otra imagen; el resultado es una superposición restringida de imágenes, basada en la técnica de diferencia controlada (modulada) las mismas. En algunas aplicaciones en las que se necesite mezclar imágenes, esta superposición restringida de imágenes podría ser una opción a considerar.
\end{abstract}

Palabras clave. procesamiento digital de imágenes, visión cibernética, visión por computadora, imágenes, fusión, diferencia, superposición.

\section{THRESHOLDED OVERLAPPING OF IMAGES}

\section{ABSTRACT}

An algorithm to select a set of pixels above a predefined grey-level threshold from an image, extract it and transfer it to another image is presented; the result is a restricted overlapping of images, based on the Controlled (modulated) image difference technique. In some applications this thresholded overlapping may be an option to consider when the aim is the mixture of images.

Keywords. Digital Image Processing, Cybernetic Vision, Computer Vision, Imaging, fusion, Difference, overlapping

\section{INTRODUCTION}

Sometimes it is necessary to combine information from different image sources in a single image; one of the techniques to achieve this combination is Image Fusion. The objective of image fusion is to combine two or more images into a single one, which as a result includes information contained in the individual component images. The resulting image from the fusion process is therefore more useful to the human eye or to computer vision. The critical point in the fusion of two or more images is the development of an algorithm for achieving their optimal combination so as to generate a useful fused image.

There is a variety of image acquisition techniques ${ }^{(1-6)}$ and every one of these techniques exhibit diverse characteristics, such as type of degradation, salient features, texture properties etc. Different imaging techniques like tomography, ultrasound, nuclear magnetic resonance, etc, may be used to obtain different imaging from the same object, and sometimes it is desirable to include the results of one technique into those of another, this is, to mix two different results in a single one.

Different illumination types (like Frontal, Rear, Diffuse, etc), produce different images of the same object, also different radiations (x-rays, gamma rays, visible, etc) produce different images of the same object. In some cases it is necessary to obtain the combination of two (or more) of these images.

In this paper, an algorithm to select a set of pixels above a predefined threshold from an image, extract it and transfer it to another image is presented. This algorithm, based on the Controlled Image Difference ${ }^{(7-8)}$, generates an overlapped image, something different to image fusion because this implies a sort of chemical combination of two images, and sometimes this has its drawbacks as it will mentioned further on.

Overlapping regions of two images might be considered a kind of copy-and-paste between two images. The region to be copied is selected above a given grey-level threshold in the donor image. Since grey levels above a given threshold may be anywhere in an image, this operation is not space-limited in the contributor image. 
The technique of modulated or controlled image difference ${ }^{(7-8)}$ allows the observation and assessment of the difference -within a specified range- between two images of the same scene or object, which have been obtained by different methods (different radiations, illumination types, etc)

In medical imaging, radiations like $x$-rays, gamma rays and others are being used; these may produce different images of the same object, and under some conditions it may be necessary to make a graduated or controlled overlapping of them.

In what follows, a panoramic view of image fusion and related themes is presented; this will be followed by the introduction of the thresholded overlapping of images algorithm.

\section{APPLICATIONS OF IMAGE FUSION}

In general, the applications of image overlapping are similar to those of image fusion.

Some known applications of image fusion are medical diagnosis, surveillance and security systems, remote sensing, concealed weapon detection, biometric systems, non-destructive evaluation, defect inspection, blurred image restoration, etc.

\section{SENSORS IN IMAGE ACQUISITION}

Sensors generate images and these may be produced using different types of sensors. In many applications a single sensor cannot provide enough information about a scene. As an example consider the case of an infrared camera detecting a hot body in a scene, this information must be complemented by a visible light photograph of the same scene, so that the hot spot can be geometrically located in the scene. Ideally the two images must be fused in a single one. Some examples of sensors producing images are optical cameras, millimetre wave cameras, infrared cameras, x-ray imagers, and radar imagers.

Since images to be fused are to be superimposed, the sensors used for image fusion need to be accurately aligned so that their images will be in spatial registration. If this is not possible, then registration algorithms are to be applied before image fusion.

\section{MAIN DRAWBACK OF IMAGE FUSION}

The most common algorithm for fusing images is the one based on pixel averaging, however this has the undesired effect of suppressing highlighting image features, because it generates a low contrast image with a rather colorless (washed-out, exhausted) appearance $^{(10)}$. Some attempts have been made to overcome this drawback ${ }^{(10)}$.

Notwithstanding image fusion has its drawbacks, since it achieves the combination of two different images, it becomes a very powerful image processing technique, with plenty of critical applications and hence very much research is being carried out on it (10-12). The technique of image fusion has proven to be very useful in medical and in remote sensing imaging.

\section{MULTISENSOR IMAGE FUSION}

Multisensor Image Fusion is a relatively new implement in the toolbox of Computer (Cybernetic) Vision dealing with the combination of two or more different-source images in a single one containing much more information than the individual component images.

\section{THE ALGORITHM TO ACHIEVE THRESHOLDED OVERLAPPING OF IMAGES}

Given two grey-levelled images of the same scene but obtained by means of different acquisition techniques, detect their difference. Set a grey-level threshold corresponding to the region of interest in one image. Perform a scanning of the image containing the region of interest and extract those pixels above the threshold and place them on the other image, in their corresponding positions. This algorithm preserves highlighting image features and for some applications may be considered an alternative to image fusion. 

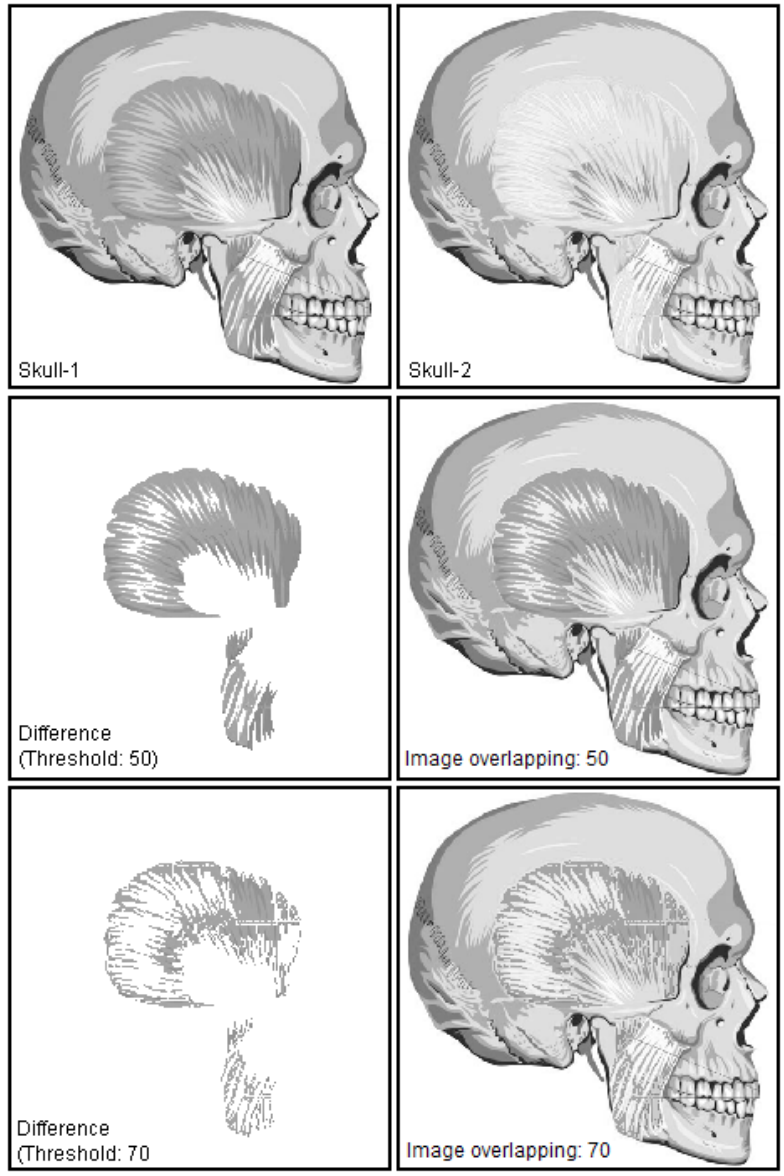

Fig. 1 Top: Two images (Skull-1 and Skull-2) of the same object but obtained with different methods. The second row shows at the left, the pixels for a threshold difference of 50 , and at the right side, the corresponding image overlapping is displayed. The bottom row shows the pixels and overlapping for a threshold of 70 . As it can be seen, resulting overlapped images include modulated (selected) information from both input images.

\section{ASSESSING THE ALGORITHM}

With the aim on assessing the algorithm of thresholded overlapping of images, two images of the same scene but obtained by different means, hence having different information, were used. In order to generate these images, a colour synthetic image of the human skull showing some muscles was converted to grey-scale by means of two methods ${ }^{(9)}$, NTSC 56-33-11 and 3-6-1. The resulting grey-level images are named

Skull-1 and Skull-2 respectively and they are shown in the top of Fig.1. These two grey-level images correspond to the same scene (or "cut" in the language of medical imagery), but are different in the sense that any of the two images include some information not present in the other. This may be appreciated in the histograms (Fig. 2) of the two original images and those of the resulting images.

The difference( ${ }^{(8)}$ in grey-levels between Images Skull-1 and Skull-2 ranges from 1 to 95, which theo- retically means that $95-1=94$ new images may be created by a thresholded overlapping of Skull-1 and Skull-2. In the second row of Fig. 1 the difference between Skull-1 and Skull-2 for a threshold of 50 is shown at the left side, and at the right side, the corresponding overlapped image appears. The bottom row of Fig. 1 displays the pixels and corresponding overlapped image for a threshold of 70 . It is not superfluous to mention that these resulting overlapped images contain selected information extracted from both original images. Analyzing the histograms in Fig.2 it can be seen that effectively, the resulting overlapped images contain information not present in the original ones.
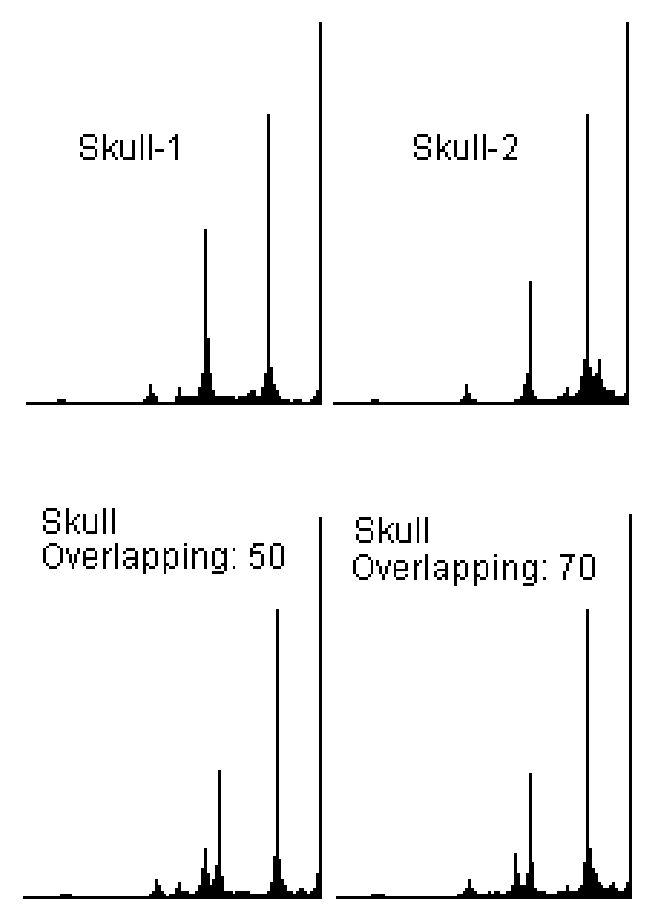

Fig 2. Histograms in the range 0 to 250. Grey levels above 251 are too highly populated and they have been discarded so as to avoid relative poor representation of levels with minor populations.

Notice in the bottom row of Fig. 1, that a horizontal line above the eye sockets shows up, this line is not present in the input images (at Fig. 1 top). After a careful revision of the original colour image, this line was identified as a set of very light variations in pixel colours. This finding will be the matter of further investigation, because it suggests the possible use of the modulated difference technique, to detect hidden details in an image. 
As must be expected from an algorithm of this type, when the difference threshold is set to its minimum, the result of the thresholded overlapping of the two images is strictly one of them, and when this threshold is set to its maximum, the result is the other image. When image overlapping is carried out under other difference threshold, the resulting image is a modulated overlapping of the input images.

\section{CONCLUSIONS}

An algorithm to carry out overlapping of two greylevelled images above a predefined grey-level has been devised, implemented and successfully tested. This algorithm may be considered as an alternative in some applications where image fusion is not the optimal technique to be applied.

It may be said that thresholded overlapping of images generates a controlled mixture of images, whereas image fusion generates a chemical combination of two images.

The algorithm here reported has been built as a Virtual Lab, so that students of Digital Image Processing may use this software tool to practice with their own images.

\section{REFERENCES}

[1] Gonzales R, Woods R, (1993), Digital Image Processing (Addison-Wesley, USA.

[2] Jahne B, (1995) Digital Image Processing, Springer-Verlag, USA.
[3] Gonzales R, Wintz P, (1987), Digital Image Processing, Addison-Wesley, USA.

[4] Schalkoff R. J, (1989), Digital Image Processing and Computer Vision Willey, USA.

[5] Chen C.H., Pau L. F, Wang P.S.P., (1995) Handbook of Pattern Recognition \& Computer Vision, World Scientific, USA

[6] Jhane B., (2004), Practical handbook on image processing for scientific and technical applications, CRC Press, USA.

[7] Montenegro Joo J, (2012) Virtual Lab for Thresholded Image Difference, submitted to Industrial Data.

[8] Montenegro Joo J, Modulated Image difference, Imagery Virtual Lab. (VirtualDynamicsSoft, 2006)

[9] Montenegro Joo J, (2009) Imagery 37: Digital Image Processing Virtual Lab., V International Conference on Multimedia and ICT in Education (m-ICTE 2009), Lisboa, Portugal.

[10] Mitchell, H. B. (2010), Image Fusion: theories, techniques and applications, SpringerVerlag, Germany.

[11] Stathaki, T. (2008) Image Fusion: Algorithms and Applications, Elsevier, The Netherlands.

[12] Blum R.S - Zheng Liu, (2006) Multisensor Image Fusion and its Applications, Taylor \& Francis, USA. 